\title{
Letter to the Editor regarding "A quantitative and comprehensive method to analyze human milk oligosaccharide structures in the urine and feces of infants"
}

\author{
Viktoria Dotz • Clemens Kunz
}

Received: 13 May 2013 / Accepted: 13 June 2013 / Published online: 16 July 2013

(C) Springer-Verlag Berlin Heidelberg 2013

To the Editor:

With great interest, we read the article by De Leoz et al. in Anal Bioanal Chem 2013 [1]. Although Lebrilla's group is one of the most respected in the field of human milk oligosaccharide (HMO) analysis, unfortunately, this publication is lacking some essential information concerning the quantitation of HMO by nano-LC chip/TOF MS:

1. The authors claim the reproducibility and precision of the nano-LC MS to be higher than those of MALDI MS, but only show precision data from MALDI measurements (Table 1).

2. Although quantitation is described to be done by means of deuterated HMO standards, no concentrations or H/D ratios, but only ESI-TOF MS abundances, are shown (Table 3). Using the expression "quantitation" in this context might possibly bear the risk that wrong conclusions are drawn by some readers.

3. Owing to the well-known phenomenon of ion suppression in ESI-MS, which is not compensated by the use of deuterated standards (cf. Duncan [2]), the quality of calibration should be addressed in addition to precision data from the quantifying instrument.

4. The issue of strongly varying water content and, thus, HMO concentrations in urine and feces is not addressed.
Therefore, the authors should comment on the practicability of the presented method to obtain true concentrations.

5. As milk, urine, and feces are very complex and distinct sample matrices and, furthermore, numerous preparation steps are required, it seems necessary to present data on analyte recovery, confirming the comparability of the three sample matrices.

Since the authors do not state the time required for sample preparation and analysis, one can hardly imagine the presented method being 'rapid high-throughput' after several time-consuming steps, such as Folch extraction, ethanol precipitation, reduction, and two-step solid phase extraction.

We would appreciate a short response from the authors.

\section{Reference}

1. De Leoz LA, Wu S, Strum JS, Niñonuevo MR, Gaerlan SC, Mirmiran M, German JB, Mills DA, Lebrilla CB, Underwood MA (2013) A quantitative and comprehensive method to analyze human milk oligosaccharide structures in the urine and feces of infants. Anal Bioanal Chem 405:4089-4105

2. Duncan MW (2012) Good mass spectrometry and its place in good science. J Mass Spectrom 47:795-809 